\title{
Beiträge zur Kenntnis und Beurteilung der Obst- und Beerenweine besonders des Apfelweins.
}

\author{
Von
}

\author{
H. Willeke und W. Schellens. \\ Mitteilung aus dem Chemischen Laboratorium der Kgl. Auslandsfleischbe- \\ schaustelle Frankfurt a. M.
}

(Eingegangen am 15. Juni 1913.)

Die Obstweine sind durch das Weingesetz rom 7. April 1909 als weinähnliche Getränke defimiert worden und unterliegen als solche nur den Bestimmungen des $\$ 10$ dieses Gesetzes und den dazu vom Bundesrat erlassenen Ausführungsbestimmungen. Die durch das Weingesetz eingeführte Bucbkontrolle ermöglicht nun den Fälschungen von Wein unter Umständen hierdurch bei den Produzenten auf die Spur zu Fommen oder sie im Verdachtsfalle auch zu beweisen. In den seltensten Fällen wird dies aber bei den Herstellern von Obstweinen möglich sein, da der Verpflichtung, die im Gesetz vorgeschriebenen Bücher zu führen, nur diejenigen Obstweinhersteller unterliegen, welche gleichzeitig Traubenwein herstellen. Letzterer Fall wird, abgesehen von Ausnahmen in weinbautreibenden Gegenden, wohl selten sein. Dagegen müssen alle diejenigen, welche Traubenwein und Obstwein gewerbsmäßig in den Verkehr bringen, nebən der Buchführung über Traubenwein-Einkauf und -Verkauf diese Buchführung auch auf den Obstwein ausdehnen. Die Zahl dieser Klein- und Großhändler, Wirte usw. ist natürlich auBerordentlich groß. Durch diese Buchkontrolle wird also Mengenvermehrungen der Obstweine bei den Händlern und Wirten möglicherweise nachzugehen sein. Bei den Herstellern ist dagegen eine solche Möglichkeit durch Feststellung der Mengen des verarbeiteten Obstes und Zuckers sowie der verkauften Mengen fertiger Erzeugnisse nicht gegeben, weil, wie gesagt, für die Mehrzahl eine Buchführungspflicht nicht besteht. Im Interesse einier besseren Aufklärung beobachteter oder vermuteter Fälschungen wäre eine solche sicher erstrebenswert, und die reellen Fabrikantenkreise werden sich auch kaum dagegen erklären, weil erst dann vielfachen Unlauterkeiten mit vollem Erfolge entgegengetreten werden kann.

Die Nahrungsmittelkontrolle ist also bei der Beurteilung solcher Obstweine auf die chemische Analyse und auf die Bestimmungen des Nahrungsmittelgesetzes angewiesen.

Der Verbrauch von aus Fruchtsäften hergestellten alkoholarmen, weinähnlichen Getränken ist uun zum Teil infolge der stets wachsenden Antialkoholbewegung zum Teil auch infolge der steigenden Weinpreise und der häufigen schlechten Weinernten zweifellos andauernd gestiegen; auch ist die Wirkung des neuen Weingesetzes, welches die früheren, ganz billigen Traubenweine vollständig zum Verschwinden gebracht hat, ebenfalls fördernd für den Absatz von Obstwein, besonders Apfelwein gewesen. Daher hatte $\mathrm{Ku}$ lis ch vollkommen Recht, wenn er in einem im Jahre 1910 in Eisenach gehaltenen Vortrage ausführte: „Es wird zu erwarten sein, daß sich durch die strengeren Vorschriften über den Verkehr mit Wein auch die Ansichten der Sachverständigen über den Obstwein diesen Vorschriften in dem Sinne anpassen werden, daß man eben nach allen Richtungen etwas strenger urteilen wird." Daß dies letztere unbedingt erforderlich ist, haben wir in Frankfurt, der Stadt des Apfelweins, reichlich 
zu beobachten Gelegenheit gehabt. Die Nahrungsmittelkontrolle dürfte danach zweifellos diesen Produkten künftig ganz besondere Aufmerksamkeit zu widmen haben. Unsere eigenen Erfahrungen bei der Nahrungsmittelkontrolle und ebenso die gleichgerichteten Anregungen von beteiligten Interessentenverbänden lassen ein schärferes Vorgehen gegen die Auswüchse schlimmster Art, wie sie sich bei diesen Fabrikationszweigen ausgebildet haben, als ein dringendes Erfordernis erscheinen. Von den Anregungen aus Interessentenkreisen seien hier einige genannt: Der Elsaß-Lothringische Verein der Obstweininteressenten ist $z$. B. angeblich ausdrücklich zu dem Zwecke gegründet worden, Klarheit über die Materie der Obstweine zu schaffen und gegen die unlauteren Machenschaften der Konkurrenz streng einzuschreiten. Der Verband der Deutschen Obst- und Beerweinkeltereien (Sitz Frankfurt a. M) bemüht sich ebenfalls auf Anregung von Prof. H. Becker Material zu sammeln, um über geeignete Unterlagen für ein von manchen Seiten gefordertes Obstweingesetz $z u$ verfügen. Daß solche Unterlagen bei weitem noch nicht in genügendem Mabe vorhanden sind, ist in der Praxis von uns ebenso wie von zahlreichen anderen Stellen, auch von reellen Fabrikanten, oft lebhaft beklagt worden. Wir haben es daher unternommen, alljährlich eine möglichst große Anzahl von selbst hergestellten oder unter unserer Aufsicht in einwandfreien Betrieben gewonnenen Obstmosten, besonders Äpfelmosten aus den verschiedensten Sorten und von der verschiedensten Herkunft zu untersuchen und die Ergebnisse zu veröffentlichen (vergl. die Tabellen III, IV und V). Wir möchten wünschen, daß sich möglichst zahlreiche Fachgenossen in Gegenden, in denen die Obstweinbereitung eine Rolle spielt, unserem Vorgehen anschließen, damit auf diese Weise neben der Traubenwein- und Fruchtsaft-Statistik auch eine Obstmost-Statistik allmählich geschaffen werden kann. Eine solche mit weiteren Untersuchungen über die Säureverhältnisse dieser Getränke, besonders über den Säurerückgang würde dann sicherlich bessere Unterlagen für ihre Beurteilung bieten, als wir sie jetzt besitzen. Derartige Untersuchungen über den Säurerückgang bezw. die Verhältnisse der einzelnen Säuren in den Obstweinen, besonders Äpfelweinen, wie sie neuerdings von H. Becker mitgeteilt wurden, sind auch von uns bereits vor Jahren begonnen worden, mußten aber wie andere aus Mangel an Zeit verschoben werden. Einige diesbezügliche Untersuchungsergebnisse, die sich übrigens mit den von $\mathrm{H}$. Becker gefundenen im großen und ganzen decken, können wir bereits jetzt mitteilen; sie sind in den beigefügten Tabellen I und II enthalten. Diese Arbeiten sollen indessen ebenfalls in größerem Umfange fortgesetzt und dann später im Zusammenhang gewürdigt werden.

Wie auBerordentlich verschieden die Ansichten bei der Beurteilung von Obstweinen sind, sei an einigen Beispielen gezeigt, die teilweise eigener Erfahrung bei der Nahrungsmittelkontrolle bezw. am Gericht entstammen, teilweise sonstigen Veröffentlichungen entnommen, sind, z. B. dem Verbandsbericht für 1910 des Verbandes der Deutschen Obstweinfabrikanten:

1. Gleiche Teile sogenannten Frucht-Muttersaftes und Zuckerwasser gemischt sollen ohne weitere Gärung einen prima Obstwein besonders für Frauen und schwäch liche Personen ergeben. Dieses Rezept wurde vom Beschuldigten am Gericht auf die Anweisung einer Weinbauschule zurückgeführt.

2. Eine Behörde soll die Ansicht vertreten haben, eine Vèrdünnung mit Zuckerwasser bis zu 25\% dürfe bei Äpfelwein anstandslos stattfinden, doch müsse analog den. Forderungen bei Traubenweinen diese Zuckerung ev. deklariert werden.

3. Ein bekannter, auf dem Gebiete der Weinchemie besonders tätiger Chemiker 
soll die Meinung ausgesprochen haben, „da隹 eine gesetzliche Beschränkung der Zuckerung für Äpfelwein überhaupt nicht gegeben sei. Man könne getrost ein Gemisch aus $25 \%$ Äpfelmost und $75 \%$ Zuckerwasser noch als Äpfelwein unbeanstandet verkaufen." - Man kann sich der hierzu von H. Becker ${ }^{1}$ ) geäußerten Ansicht sicherlich anschließen, daß nämlich solche Produkte nicht mehr als gezuckerter Äpfelwein sondern als "geäpfeltes Zuekerwasser" anzusehen seien.

4. Der Leiter einer önochemischen Versuchsstation vertritt folgenden Standpunkt: „daß ... eine Vermehrung des Äpfelweins um mehr als $20 \%$ niemals notwendig ist und deshalb stets als eine Verfälschung betrachtet werden muf.... Ob nicht unter gewissen Bedingungen schon eine geringere Streckung technisch eine Verfälschung darstellt, dürfte sich hauptsächlich nach dem Säuregehalt richten. Unterschreitet in einem Äpfelwein der Säuregehalt 3-4\% (soll natürlich \%oo heißen), so liegt, falls eine Streekung vorgenommen worden ist, ebenfalls eine Fälschung vor, denn die Streckung war hier technisch nicht notwendig."

5. Definitionen der Obstweinfabrikanten-Verbände aus neuester Zeit lauten folgendermaßen :

Äpfelwein (in einigen Gegenden Deutschlands entgegen dem sonst üblichen Sprachgebrauch "Äpfelmost" und Most) ist das durch alkoholische Gärung aus dem Safte der frischen Äpfel hergestellte Getränk. Ein angemessener Zusatz von Wasser und technisch reinem Rüben-, Robr-, Invertzucker ist unter Umständen je nach der Eigenart und Reife des verwendeten Obstes während der Kelterzeit geboten und zulässig. Keinesfalls soll der Zusatz von Wasser mehr als 25\% des Mostes, das ist 20\% des ganzen Gemisches betragen. Naturreiner Apfelwein, der als solcher verkauft und feilgehalten wird, darf keinerlei derartige Zusätze erhalten.

Birnwein wie Apfelwein.

Beerenweine sind die durch alkoholische Gärang aus dem Safte friseher Beeren unter Zusatz von Wasser und technisch reinem Rüben-, Rohr-, Invertzucker hergestellten Getränke. Ein Zusatz von reinem Sprit zu Beerenwein ist erlaubt und unter Umständen sogar notwendig. Der Spritzusatz darf nicht über 3 Gewichtsprozent betragen. Eine Zusammensetzung aus Sprit, Obstsaft und Wasser ohne gemeinsame Gärung der letztgenanten beiden Bestandteile ist $\mathrm{k}$ ein Fruchtwein und darf als solcher nicht verkauft werden. Verboten ist die Herstellung von Fruchtwein aus vergorenen oder konservierten Obstsäften unter Zusatz von Wasser und Zucker durch nachträgliche Vergärung. Für die Kellerbebandlung der Fruchtweine sind dieselben Grundsätze masggebend wie für die Kellerbehandlung des Weines.

Süfivergorener (sülier) Äpfelwein ist das durch alkoholische Gärung aus dem mit Zucker versetzten Safte der frischen Äpfel hergestellte Getränk. Analog dem Äpfelwein ist der Zusatz von Nachpresse gestattet. In $100 \mathrm{~kg}$ des fertigen Getränkes dürfen höchstens $15 \mathrm{~kg} \mathrm{zu}$ gesetztes Wasser enthalten sein. Ein Alkoholzusatz von $3 \%$ ebenso wie Kellerbehandlung soll analog den Vorschriften für Beerenweine gestattet sein.

6. Schließlich sei noch ein Passus aus einer neuerdings vom Reichsgericht gefällten Entseheidung ${ }^{2}$ ) hier angeführt:

„Der Tatriehter geht ersichtlich von der rechtlich nicht za beanstandenden Annahme aus, dab Erzeugnisse, die sich durch ihre Benennung und durch ihre äuf̉ere Erscheinung als ein durch alkoholische Gärung von Obstsäften gewonnenes Getränk darstellen, durch Wasserzusätze nicht oder doch nur in beschränktem Maße verdünnt werden dürfen. Fordert die

1) Verbandsbericht für 1910 des Verbandes der Deutschen Obstweinfabrikanten.

$\left.{ }^{2}\right)$ Gänther, Sammlung von Entscheidungen der Gerichte auf Grund des Weingesetzes. 1913 , Heft II, S. 59 . 
Verkehrsauffassung, wie der Tatrichter annimmt, nicht Naturreinheit der Obstweine, namentlich des Äpfelweines, sondern läßst sie Wasserzusätze in der Höhe von $10 \%$ der Menge des Obstsaftes zu, vorausgesetzt, daß der Zusatz zur Auslaugung der Trester erfolgt - eine Feststellung, die zwar im vorliegenden Falle bindend ist, deren Richtigkeit aber dahinstelt so ist es zutreffend als Verfälschang erkannt, wenn gröGere Zusätze als der angegebene and ohne Rücksicht darauf stattfinden, ob die Zusätze für die Auslaugung der Trester notwendig sind, oder ob sie lediglich zum Zwecke der Vermehrung der Mienge dienen sollen oder gar erst dem gewonnenen Safte, wie er zur Gärang kommt, oder dem fertigen Weine in übermäßigiger Menge zugeführt werden. Denn durch die überschießenden Mengen Wassers, eines gegenüber den Obstsäften minderwertigen Stoffes, triţ eine Verdünnung und damit eine Ver* sehlechterung des Enderzengnisses ein, während nur die zur Auslangung der Trester bestimmten und nur mäßigen Zuckerwasserzusätze im Interesse dex Äpfelweinbereitung liegen und deshalb vom Publikum erwartet und gebilligt werden können. Gerade darin, daß fremde und minderwertige Stoffe dem fertigen Erzeugnis oder auch schon während der Herstellung des Zwischenerzengnisses zugesetzt werden, besteht das Wesen der Verfälschung".

Diese letzte Beurteilungsnorm unseres höchsten Gerichtes dürfte zweifellos sowohl für die Mehrzahl der reellen Hersteller wie vor allen Dingen für die Verbraucher die ersprieblichste sein, und es wäre zu wünschen, daß sie möglichst allgemein. Anerkennung und vor allen Dingen Anwendung fände.

Gerade das letztere hat seine großen Schwierigkeiten, die eben in der Unsicherheit der Bewertung der analytisch gefundenen Zahlen liegen. Darin ist den Angaben Bujard und Baier's in ihrem Hilfsbuch für Nahrungsnittelchemiker beizupflichten, wenn sie angeben:

Uber die Zusammensetzung der Obstweine lassen sich Werte nicht angeben, da die. Schwankungen sehr groß sind; bei Apfel- und Birnenwein sind letztere im allgemeinen geringer als bei den Beerenweinen, Infolge eines meist großen Säuregehaltes muß bei Beerenweinen oft eine erhebliche Streckung mit Zuekerwasser vor der Vergärung vorgenommen. werden. Der Zucker- und Säuregehalt der Äpfel und Birnen erfordert einen solchen Zusatz jedoch nicht. Die noch vielfach gemachten Wasserzusätze (lediglich Verdünnungen) bei derartigen Obstweinen sind daher als Verfälschung im sinne des $\$ 10$ des Nahrungsmittelgesetzes. anzusehen. Der Extraktgehalt unverdünnter Äpfelweine beträgt etwa 2,5 g, der Aschengehalt etwa 0,25 g. Der Alkoholgehalt $5-6 \mathrm{~g}$ in $100 \mathrm{ccm}$. Nach allgemeinen Grenzzahlen lassen. sich Verfälschungen nicht nachweisen.

Der letztere Satz ist ganz besonders hervorzuheben, denn die angegebenen Zahlen bieten tatsächlich nur ganz annähernde Durchschnittswerte und dürfen allein keinesfalls zur Beurteilung dienen, vielmehr wird der Hauptwert auf die Verhältnisse der Säuren zu legen sein. Dabei wird man aber davon auszugehen haben, daß nicht vollständig unreifes Obst mit außerordentlich viel Säure das normale Rohmaterial solcher Weine bildet. Denn $\mathrm{n}$ ur bei Verwendnng erbeblicher Mengen unreifer Äpfel ist tatsächlich, wie unsere Erfahrung gelehrt hat, ein irgendwie erheblicher Zuckerwasserzusatz erforderlich. $\mathrm{Ob}$ im Einzelfalle ein Zuckerwasserzusatz "geboten" und ob ein solcher "angemessen" war, wird daher schwer festzustellen sein, da die Eigenart und die Reife des verwendeten Obstes nachträglich am fertigen Erzeugnis nicht mehr zu erkennen ist. Gerade nach dieser Richtung sind die bisher bekannten Unterlagen für die Beurteilung des Äpfelweins keineswegs ausreichend, und es muß daher das Bestreben aller an diesen Fragen beteiligten Kreise, besonders aber der amtlichen Nahrungsmittelkontrolle, sein, die beteiligten Interessenten in ibren lobenswerten Reellitätsbestrebungen zu unterstützen und zur Klärung dieser Fragen durch Schaffung geeigneter, statistischer Unterlagen beizutragen. Die Anfänge zu solchen sind in den nachstehenden Untersuchungsergebnissen von teils selbsthergestellten Obst- 
säften, teils aus renommierten Obstweinkeltereien entnommenen Proben enthalten. Ein besonderer Wert dürfte, wie oben bereits erwähnt, auf die Veränderungen zu legen sein, welche alle diese weinähnlichen Getränke nach dem Verlaufe der stürmischen Gärung und auch vielfach noch nach Jängerem Lagern erleiden. Gerade diese Veränderungen der Säuren, welche ja auch beim Traubenwein eine große Rolle spielen, sind anscheinend bisher bei den verschiedenen Obstweinen noch gar zu wenig gewürdigt, und es ist daher eine weitere Vertiefung der Kenntnis dieser Verbältnisse für eine wirklich einwandfreie Beurteilung solcher Erzeugnisse einer blühenden Industrie eine wichtige Vorbedingung.

Tabelle I.

1911-er Äpfelmoste (a) und daraus hergestellte Äpfelweine (b) einer bedeutenden Äpfelweinkelterei; zur Veranschaulichung der Veränderung der Sänren.

\begin{tabular}{|c|c|c|c|c|c|c|c|c|c|c|c|c|c|c|c|}
\hline \multirow[t]{2}{*}{$\stackrel{0}{Z}$} & \multirow[t]{2}{*}{ Bezeichnung } & \multirow[t]{2}{*}{$\mid \begin{array}{c}\text { Spezi- } \\
\text { fisches } \\
\text { Ge- } \\
\text { wicht }\end{array}$} & 司 & 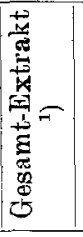 & 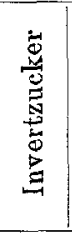 & 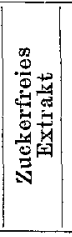 & 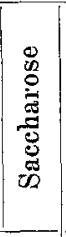 & 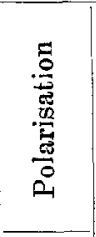 & 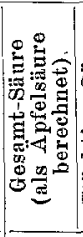 & 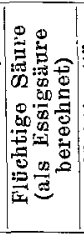 & 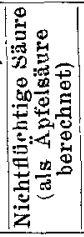 & 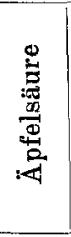 & 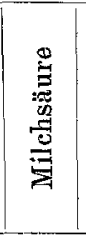 & $\frac{8}{0}$ & \multirow{2}{*}{ 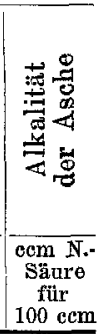 } \\
\hline & & & \multicolumn{5}{|c|}{$\mathrm{g}$ in $100 \mathrm{ecm}$} & $\begin{array}{c}\alpha_{\mathrm{D}}^{\mathrm{im}} \\
100 \mathrm{~mm}- \\
\mathrm{Rohr}\end{array}$ & \multicolumn{6}{|c|}{$\mathrm{g}$ in $100 \mathrm{cem}$} & \\
\hline \multirow{2}{*}{1} & Aus dem Taunus; (a & 1,0463 & - & 11,99 & 7,72 & 27 & - & $-3,20$ & 0,556 & 0,024 & 40,529 & - & 0,054 & 0,320 & 2,7 \\
\hline & $\begin{array}{c}\text { mit spelerling; ohne } \\
\text { Wasserzusatz }\end{array}$ & 1,0009 & 3,46 & 1,97 & 0,03 & 1,94 & - & 0 & 0,422 & 36 & 0,382 & - & 0,290 & 0,272 & 2,5 \\
\hline \multirow{2}{*}{2} & $\begin{array}{l}\text { Aus dem Taunus; } \\
\text { mit Speierling und a }\end{array}$ & 0445 & 0,37 & 11,70 & 8,17 & 3,53 & - & $-3,32$ & 0,482 & 0 , & 40,455 & - & 0,100 & 0,268 & 2,7 \\
\hline & Reinhefe; gezuckert b & 1,0007 & 4,59 & 2,23 & 0,03 & 2,20 & - & 0 & 0,292 & 0 & 0,246 & - & 0,198 & 0,254 & 2,7 \\
\hline \multirow{2}{*}{3} & Aus dem T & 1,0477 & - & 12,35 & 7,85 & 4,50 & - & $-3,52$ & 0,598 & 0,017 & 70,574 & - & 0,073 & 0,280 & 2,5 \\
\hline & hefe; ohne Zucker (b & 1,0024 & 4,41 & 2,39 & 0,04 & 2,85 & - & 0 & $0,335 \mid$ & 10,050 & 0,281 & - & 0,240 & 0,278 & 2,45 \\
\hline \multirow[t]{3}{*}{4} & $\begin{array}{l}\text { Speierling; mit Zu- } \\
\text { satz von Reinhefe }\{\text { a }\end{array}$ & 1,0324 & 1,39 & 9,05 & 5,48 & 3,57 & - & $-3,08$ & 0,610 & 0,026 & 0,581 & - & 0,062 & $0.272 \mid$ & 2,9 \\
\hline & und Zucker |b & 1,0013 & 4,35 & 2,31 & 0,04 & 227 & -1 & 0 & {$[0,308$} & $8 \mid 0,060$ & 0,241 & 一 & 0,200 & $0,258 \mid$ & 2,5 \\
\hline & $\begin{array}{l}\text { Äpfe } \\
\text { nach }\end{array}$ & & u & 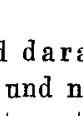 & Tab & 1 & & M & & & . & uent & (c). & en & \\
\hline \multirow{3}{*}{1} & & 1,0476 & 0,16 & $12,40 \mid$ & 9,67 & 2,73 & 1,93 & - & & & & 0,593 & 0,072 & $|0,288|$ & 2,4 \\
\hline & - & 1,0013 & 4,11 & 2,29 & 0,26 & 2,03 & - & - & 0,375 & 0 & 70,305 & 0,127 & 0,225 & 0,272 & 2,7 \\
\hline & & 1,0005 & 4,15 & 2,02 & 0,21 & 1,81 & - & - & 0,408 & 0,077 & 70,328 & 0,110 & 0,240 & 0,256 & 2,7 \\
\hline \multirow{3}{*}{2} & a & 1,0509 & 0 & 13,29 & 10,20 & 3,09 & 1,97 & - & & & 0,543 & 0,612 & 0,067 & 0,288 & 2,5 \\
\hline & - & 1,0013 & 5,08 & 2,34 & 0,31 & $\mid 2,03$ & - & - & 0,402 & & & 0,17 & & 280 & 2,8 \\
\hline & c & 1,0010 & 5,08 & 2,22 & 0,25 & 1,97 & - & - & 0,388 & $30 ; 120$ & 0,254 & 0,121 & 0,250 & 0,268 & 2,9 \\
\hline \multirow{3}{*}{3} & & 1,0568 & 0,16 & 14,80 & 11,00 & 3,80 & 2,15 & - & 0,844 & 0,012 & 0,831 & 0,897 & 0,060 & 0,400 & 4,0 \\
\hline & - & 1,0066 & 5,01 & 3,67 & 1,06 & 2,61 & - & - & 0,442 & & 50,217 & 0,201 & 0,203 & 0,372 & 4,5 \\
\hline & & 1,0025 & 5,14 & 2,77 & 0,17 & 2,60 & - & - & 0,482 & 0,092 & 0.379 & 0,087 & 0,265 & 0,344 & $=4,3$ \\
\hline \multirow{3}{*}{4} & & 1,0431 & 0,47 & 11,31 & 8,90 & 2,41 & 1,49 & - & 1,010 & $\overline{0,026}$ & 60,981 & $\overline{1,140}$ & 0,060 & $\overline{0,372}$ & 3,8 \\
\hline & 一 & 1,0040 & 4.41 & 2,73 & 0,15 & 2,58 & - & - & 0,750 & 0,056 & $6 \mid 0,687$ & 0,683 & 0,171 & & 3,8 \\
\hline & & 1,0028 & 4,59 & 2,22 & 0,10 & 2,12 & - & - & 0,509 & 9,060 & 0,442 & 0,147 & 0,305 & 0,312 & $\begin{array}{l}3,8 \\
\end{array}$ \\
\hline \multirow{3}{*}{5} & & 1,0453 & 0,26 & 11,86 & 8,62 & $\overline{3,24}$ & 0,79 & - & & & & $\overline{1,030}$ & & $\overline{0,356}$ & 3,6 \\
\hline & - & 1,0044 & 4,41 & 2,84 & 0,33 & 2,51 & - & - & 0,857 & 80 & 0,809 & 0,649 & 0,110 & $|0,304|$ & 3,5 \\
\hline & & 1,0028 & 4,47 & 2,21 & 0,14 & $\mid 2,07$ & $1-$ & - & 0,677 & 70,045 & $5,0,626$ & 0,402 & 0,260 & $|0,284|$ & 3,5 \\
\hline
\end{tabular}

3) Das Gesamt-Extrakt ist bei den Mosten indirekt, dagegen bei den Weinen direkt bestimmt worden. 
Tabelle III.

1911-er Fruchtweine aus einer renommierten Kelterei.

a) Sofort nach Entnahme der ganz jungen Weine untersucht. b) Dieselben Weine nach mehrmonatlicher Aufbewahrung im Laboratorium. c) Dieselben Weine etwa gleichzeitig mit $b$ untersucht, aber nach dem Lagern der Weine in den Originalfässern der Kelterei.

\begin{tabular}{|c|c|c|c|c|c|c|c|c|c|c|c|c|c|c|c|}
\hline \multirow{3}{*}{ 竞 } & \multirow{3}{*}{ Bezeichnung } & \multirow{3}{*}{\multicolumn{2}{|c|}{$\left|\begin{array}{c}\text { Spezi- } \\
\text { fisches } \\
\text { Ge- } \\
\text { wicht }\end{array}\right|$}} & \multirow{3}{*}{$\frac{\bar{a}}{\dot{8}}$} & \multicolumn{2}{|c|}{$\begin{array}{l}\text { Gesamt- } \\
\text { Extrakt }\end{array}$} & \multirow{2}{*}{ 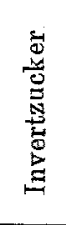 } & \multirow{3}{*}{ 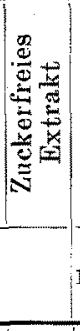 } & \multirow{3}{*}{ 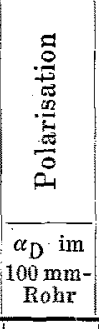 } & \multirow{3}{*}{ 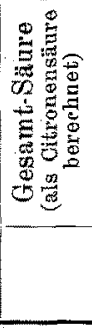 } & \multirow{2}{*}{ 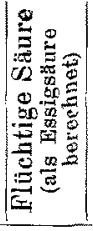 } & \multirow{2}{*}{ 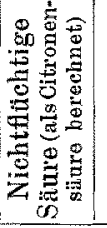 } & \multirow{2}{*}{ 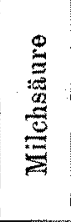 } & \multirow{3}{*}{$\frac{9}{3}$} & \multirow{3}{*}{ 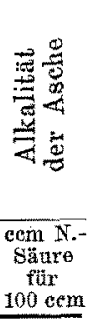 } \\
\hline & & & & & $\frac{\overrightarrow{3}}{9}$ & 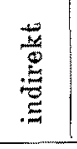 & & & & & & & & & \\
\hline & & & & & \multicolumn{3}{|c|}{$g$ in $100 \mathrm{~cm}$} & & & & \multicolumn{3}{|c|}{$g$ in $100 \mathrm{~cm}$} & & \\
\hline \multirow{3}{*}{1} & \multirow{3}{*}{$\begin{array}{l}\text { Heidelbeerwein I, } \\
\text { süßs }\end{array}$} & \multirow{3}{*}{$\begin{array}{l}a \\
b \\
c\end{array}$} & 1,0693 & 6,73 & 21,608 & 21,02 & 17,71 & 3,32 & - & 0,511 & - & - & - & 0,128 & 1,0 \\
\hline & & & 1,0602 & 7,53 & 19,301 & 18,95 & 16,40 & 2,55 & - & 0,501 & 0,094 & $0,407 \mathrm{C}$ & 0,148 & 0,104 & 0,95 \\
\hline & & & $1,0468 \mid$ & 7,87 & $16,20\}$ & 15,58 & 12,96 & 2,62 & - & 0,499 & 0,114 & 0,377 & 0,149 & 0,102 & 0,9 \\
\hline \multirow{3}{*}{2} & \multirow{3}{*}{$\begin{array}{l}\text { Heidelbeerwein II, } \\
\text { süfB }\end{array}$} & $a$ & 1,0684 & 7,53 & 21,86 & 21,10 & 18,41 & 2,69 & - & 0,556 & - & - & - & $\overline{0,108}$ & 1,0 \\
\hline & & $b$ & 1,0646 & 7,87 & 20,562 & 20,23 & 17,66 & 2,57 & - & 0,550 & 0,097 & 0,446 & 0,126 & 0,092 & 0,9 \\
\hline & & lo & 1,0480 & 8,98 & 17,14 & 16,31 & 13,81 & 2,50 & - & 0,520 & 0,110 & 0,410 & 0,108 & 0,104 & 0,9 \\
\hline \multirow{3}{*}{3} & \multirow{3}{*}{$\begin{array}{l}\text { Heidelbeerwein I, } \\
\text { herb }\end{array}$} & a & 1,0128 & 6,58 & 5,28 & 6,23 & 3,90 & 2,83 & - & 0,589 & - & -1 & 0,046 & 0,136 & 1,2 \\
\hline & & b & 1,0005 & 7,94 & 3,45 & 3,59 & 1,60 & 1,85 & - & 0,563 & 0,084 & 0,477 & 0,040 & 0,130 & 1,0 \\
\hline & & $\mathrm{co}$ & 0,9924 & 8,70 & 1,68 & 1,78 & 0,05 & 1,78 & - & 0,557 & 0,060 & 0,493 & 0,060 & 0,127 & 1,0 \\
\hline \multirow{3}{*}{4} & \multirow{3}{*}{$\begin{array}{l}\text { Heidelbeerwein II, } \\
\text { herb }\end{array}$} & 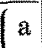 & 1,0192 & 6,40 & 7,83 & 7,52 & 5,50 & 2,33 & - & 0,698 & - & - & - & 0,160 & 1,2 \\
\hline & & b & 1,0151 & 6,73 & 6,76 & 6,90 & 4,54 & 2,36 & - & 0,672 & 0,086 & 0,580 & 0,039 & 0,160 & 1,1 \\
\hline & & 8 & 0,9940 & 9,13 & 2,22 & 2,35 & 0,09 & 2,26 & - & 0,678 & 0,065 & 0,609 & $0,045 \mid$ & 0,150 & 1,1 \\
\hline \multirow[b]{2}{*}{5} & \multirow{2}{*}{$\begin{array}{c}\text { Johannisbeerwein, } \\
\text { weif3 }\end{array}$} & $\int a$ & 1,0144 & 10,74 & 7,92 & 8,20 & 5,72 & 2,48 & - & 0,768 & - & - & - & 0,232 & 1,9 \\
\hline & & b & 1,0088 & 11,12 & 6,86 & 6,88 & 4,50 & 2,38 & - & 0,749 & 0,096 & 0,647 & 0,067 & $0,228 \mid$ & 1,7 \\
\hline \multirow{3}{*}{6} & \multirow{3}{*}{$\begin{array}{c}\text { Johannisbeerwein, } \\
\text { rot }\end{array}$} & $a$ & 1,0626 & 7,39 & 19,66 & 19,52 & 16,90 & 2,62 & - & 0,781 & - & - & - & 0,212 & 1,9 \\
\hline & & $b$ & 1,0544 & 8,07 & 18,34 & 17,64 & 15,35 & 2,29 & - & 0,749 & 0,072 & 0,672 & 0,060 & 0,200 & 1,5 \\
\hline & & $\mathrm{c}$ & 1,0224 & 10,14 & 10,25 & $10, \mathrm{C} 6$ & 7,69 & 2,37 & - & 0,736 & 0,126 & 0,602 & $\mid 0,067$ & $|0,174|$ & 1,5 \\
\hline \multirow[b]{2}{*}{7} & & $\int a$ & $\overline{1,0204}$ & 9,20 & 9,12 & 9,21 & 6,94 & 2,27 & - & 0,787 & - & - & - & 0,200 & 1,7 \\
\hline & Stachelbeerwein & (b & 1,0146 & 9,63 & 7,97 & 7,86 & 5,58 & 2,28 & - & 0,749 & 0,090 & 0,653 & 0,060 & 0,180 & 1,85 \\
\hline 8 & Stachelbeerwein & & 1,0339 & 10,07 & 13,28 & 13,08 & 10,89 & 2,14 & - & 0,672 & 0,08 & 0,582 & 0,090 & 0,176 & 1,3 \\
\hline
\end{tabular}

Tabelle IV.

1910-er und 1911er Äpfelmoste; teils selbst hergestellt, teils aus einer großen Apfelweinkelterei.

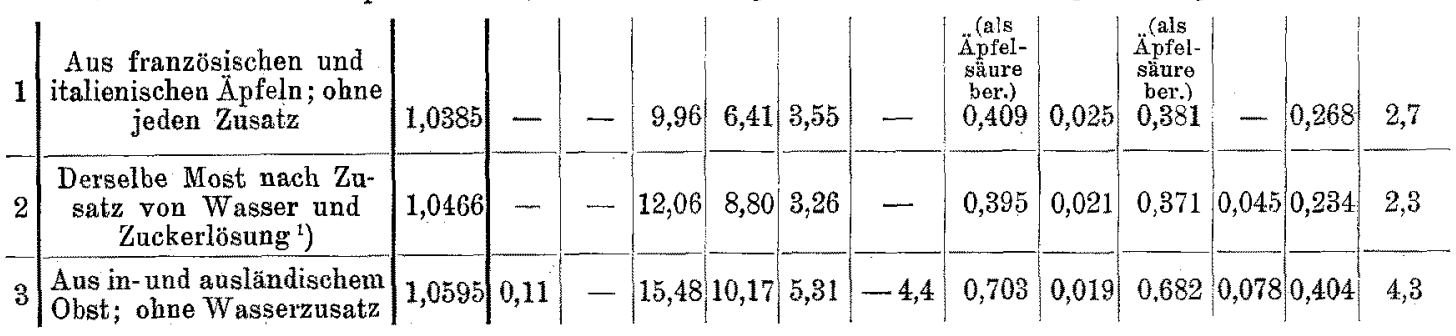

1) Nach Angabe der Firma waren zu 25 hl Most etwa 601 Zuckerlösung zugesetzt. N. 13 . 


\begin{tabular}{|c|c|c|c|c|c|c|c|c|c|c|c|c|c|c|}
\hline \multirow[t]{2}{*}{ 完 } & \multirow[t]{2}{*}{ Bezeichnung } & \multirow[t]{2}{*}{$\begin{array}{c}\text { Spezi- } \\
\text { fisches } \\
\text { Ge- } \\
\text { wicht }\end{array}$} & $\begin{array}{l}\overrightarrow{8} \\
\text { 童 } \\
\stackrel{3}{4}\end{array}$ & 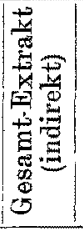 & 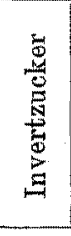 & 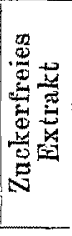 & 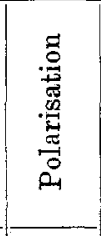 & 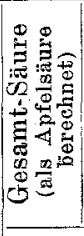 & 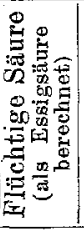 & 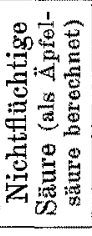 & 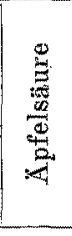 & 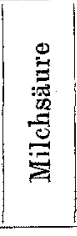 & 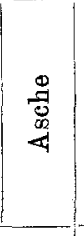 & \multirow{2}{*}{ 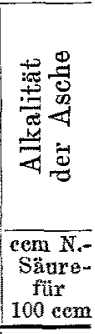 } \\
\hline & & & \multicolumn{4}{|c|}{$g$ in $100 \mathrm{cem}$} & $\begin{array}{l}\alpha_{D} \mathrm{im} \\
100 \mathrm{~mm}- \\
\text { Rohr }\end{array}$ & \multicolumn{6}{|c|}{$\mathrm{g}$ in $100 \mathrm{~cm}$} & \\
\hline 4 & $\begin{array}{l}\text { Aus in und auslän dischem } \\
\text { Obst; etwas Wasserzusatz }\end{array}$ & 1,0544 & 0,37 & 14,28 & 7,57 & 6,71 & $-3,30$ & 0,670 & 0,015 & 0,653 & 0,580 & 0,090 & 0,392 & 3,9 \\
\hline 5 & $\begin{array}{c}\text { Taunusobst } \\
\text { (ohne Wasserzusatz) }\end{array}$ & 0628 & - & 16,28 & 11,04 & 5,24 & $-4,76$ & 0,831 & 0,011 & 0,819 & 0,754 & 0,08 & 0,424 & 3,8 \\
\hline 6 & \multirow{6}{*}{ Inländisches Obst } & 1,0520 & 0,90 & 13,91 & 9,81 & 4,10 . & $-4,84$ & 0,764 & 0,040 & 0,719 & 0,687 & 0,109 & 0,392 & 3,7 \\
\hline 7 & & 1,0544 & 0,96 & 14,56 & 9,12 & 5,44 & $-4,40$ & 0,878 & 0,047 & 0,826 & 0,751 & 0,101 & 0,448 & 3,7 \\
\hline 8 & & 1,0484 & $1, \overline{71}$ & 13,36 & $\longdiv { 8 , 6 5 }$ & 4,71 & $-4,75$ & $\overline{0,643}$ & 0,019 & 0,622 & 0,522 & 0.135 & 0,492 & 4,4 \\
\hline 9 & & 1,0356 & 2,38 & 10,35 & 6,70 & 3,65 & $-4,66$ & 0,670 & 0,014 & 0,654 & 0,571 & 0,112 & 0,332 & 3,6 \\
\hline 10 & & 1,0406 & 1,33 & 11,15 & 7,74 & 3,41 & $-4,40$ & 0,590 & 0,013 & 0,575 & 0,516 & 0,079 & 0,324 & 3,1 \\
\hline 11 & & 1,0415 & 1,55 & 11,49 & 7,64 & 3,85 & $-4,51$ & 0,837 & 0,012 & 0,824 & 0,774 & 0,067 & 0,348 & 3,6 \\
\hline 12 & \multirow{5}{*}{$\begin{array}{c}\text { Selbstgekeltert im } \\
\text { Jahre } 1910\end{array}$} & 1,0460 & - & 11,21 & 6,45 & 5,41 & $-2,09$ & 0,690 & - & - & - & - & 0,280 & 3,6 \\
\hline 13 & & 1,0443 & - & 11,47 & 8,58 & 2,91 & $-3,80$ & 0,744 & - & - & - & - & 0,297 & 3,4 \\
\hline 14 & & 1,0438 & - & 11,34 & 6,37 & 4,97 & $-2,75$ & $\overline{0,489}$ & - & - & - & - & 0,222 & 2,7 \\
\hline 15 & & 1,0447 & 0,05 & 11,57 & 6,80 & 4,77 & - & 0,656 & - & - & - & - & 0,298 & 3,4 \\
\hline 16 & &, 0417 & - & 10,79 & 7,88 & 2,91 & $-3,63$ & $|0,616|$ & - & - & - & 一 & 0,255 & 3,0 \\
\hline
\end{tabular}

Tabelle V. 1912-er Äpfelmoste.

\begin{tabular}{|c|c|c|c|c|c|c|c|c|c|c|c|c|}
\hline \multirow{15}{*}{$\begin{array}{l}\text { Aus verschiedenen, } \\
\text { grofen and kleineren } \\
\text { Keltereien. }\end{array}$} & 1,0541 & 0,26 & {$[14,14$} & 10,88 & 3,26 & - & 0,781 & 0,010 & 0,770 & $|0,810| 0,080$ & 0,308 & 2,8 \\
\hline & 1,0514 & 0,32 & 13,47 & 10,01 & 3,46 & - & 0,824 & 0,030 & 0,793 & $0,8340,030$ & 0,360 & 2,9 \\
\hline & 1,0466 & $\overline{0,21}$ & 12,17 & 8,97 & 3,05 & - & 0,777 & 0,015 & 0,760 & $0,890,0,050$ & $\mid 0,328$ & 2,7 \\
\hline & 1,0455 & 0,21 & 11,88 & 9,13 & 2,75 & - & 0,569 & 0,012 & 0,556 & $0,628,0,080$ & 0,308 & 2,7 \\
\hline & 1,0480 & 0,11 & 12,48 & 10,08 & 2,40 & - & 0,576 & 0,020 & 5,554 & $0,660,0,060$ & $\overline{0,352}$ & 3,0 \\
\hline & 1,0512 & - & 13,26 & 10,31 & 3,10 & $\cdots$ & 0,549 & 0,029 & 0,518 & $\overline{0,649:-}$ & 0,320 & 2,7 \\
\hline & 1,0528 & - & 13,68 & 10,68 & 3,00 & - & 0,710 & 0,017 & 0,690 & $\overline{0,831}-$ & 0,348 & 3,2 \\
\hline & 1,0511 & 0,37 & 13,42 & 10,31 & 3,11 & - & $\overline{0,543}$ & 0,012 & 0,580 & $0,610,0,067$ & 0,292 & 2,5 \\
\hline & 1,0517 & 0,21 & 13,49 & 9,96 & 3,53 & - & $\overline{0,677}$ & 0,030 & 0,644 & $0,7900,100$ & 0,324 & 2,8 \\
\hline & 1,0466 & 0,34 & 12,22 & 9,00 & 3,22 & - & 0,985 & 0,060 & 0,918 & $1,0950,050$ & 0,308 & 3,3 \\
\hline & 1,0489 & 0,26 & 12,79 & 9,63 & 3,16 & - & 0,576 & 0,024 & 0,549 & $0,609,0,056$ & 0,384 & 3,6 \\
\hline & 1,0421 & 0,16 & 10,97 & 8,13 & 2,84 & - & 0,542 & 0,012 & 0,529 & $0,5990,060$ & 0,280 & 2,9 \\
\hline & 1,0461 & 0,20 & 12,01 & 9,40 & 2,61 & - & 0,630 & 0,040 & 0,585 & $0,7770,050$ & 0,294 & 3,3 \\
\hline & 1,0398 & 0,21 & 10,40 & 7,68 & 2,72 & - & 0,723 & 0,050 & 0,667 & $0,6900,045$ & 0,304 & 3,0 \\
\hline & 1,0372 & $\overline{0,32}$ & 9,78 & 7,30 & 2,48 & - & 0,496 & $\overline{0,012}$ & 0,483 & $0,4930,050$ & 0,280 & 2,5 \\
\hline
\end{tabular}

